\title{
ANÁLISIS DE CRECIMIENTO INICIAL DE CINCO GENOTIPOS DE BRACHIARIA EN TRÓPICO HÚMEDO
}

\author{
Autores: \\ Jonathan Raúl Garay-Martínez' \\ Eugenia Guadalupe Cienfuegos-Rivas ${ }^{*_{1}}$ \\ José Antonio Espinosa-Marroquín² \\ Pedro Zárate-Fortuna ${ }^{1}$ \\ Martín Antonio Ibarra-Hinojosa'
}





\section{Resumen}

Se condujo un estudio para evaluar el crecimiento inicial de cinco variedades de Brachiaria en la época de lluvia característica del clima tropical húmedo de la provincia de Santo Domingo de los Tásachilas, Ecuador. Los materiales genéticos evaluados fueron variedades mejoradas de Brachiaria brizantha (Piatá, Xaraés y Marandú), Brachiaria híbrido(Mulato II) y la variedad Brachiaria decumbens, considerada como testigo por ser la que prevalece en la zona en estudio. Las variedades fueron sembradas por semilla en fundas de plástico y colocadas a la intemperie para evaluar el crecimiento durante las doce semanas posteriores a la emergencia. Los resultados indican que el híbrido Mulato II tiene mayor potencial de establecimiento ( $\mathrm{P}<0.05)$ que $\mathrm{B}$. decumbens; sin embargo, cuando se estimó la tasa de crecimiento, la variedad Marandú fue superior (P<0.05) desde el inicio de la evaluación, expresado por una mayor acumulación de biomasa por unidad de área. La tasa de asimilación neta (TAN) y la tasa relativa de crecimiento (TRC) mostraron un comportamiento descendente en todas las variedades ( $\mathrm{P}>0.05)$ a lo largo del tiempo, siendo el hibrido Mulato II el que presentó la menor TAN, debido probablemente a la menor altura y mayor área foliar. Los resultados obtenidos demuestran la importancia de determinar la eficiencia fisiológica de las plantas forrajeras en el ambiente donde se van a cultivar, para garantizar la eficiencia durante el establecimiento de las praderas.

Palabras clave: Pasto, Mulato II, Marandú, decumbens, Piatá, Xaraés, área foliar, hoja, tallo, raíz.

\section{Abstract}

A study was conducted to evaluate the initial growth of five Bracharia varieties in the rainy season characteristic of the humid tropic prevalent in the Santo Domingo de los Tsáchilas Province, Ecuador. The evaluated genetic material were improved varieties of Bracharia brizantha (Piatá, Xaraés y Marandú), a hybrid (Mulato II) and the variety Brachiaria decumbens, considered the check because this is the more spread material in the region. The materials were randomly distributed in plastic bags and cultivated in the open to evaluate growth during the twelve weeks after seed emergency. Results indicate that the hybrid Mulato II has higher plant establishment potential ( $\mathrm{P}<0.05)$ than $\mathrm{B}$. decumbens. However, when the growth rate was evaluated, the Marandú variety was superior $(P<0.05)$ from the beginning of the evaluation, which translates in a greater biomass gain per unit area. The rate of net assimilation (RNA) and the relative growth rate (RGR) has a declining trend in all varieties $(\mathrm{P}<0.05)$ through time, the hybrid Mulato II being the one which presented the lower RNA, probably due to a lower height and greater leaf area. Reported data demonstrates the importance of determining plant physiological efficiency in the environment where they are going to be cultivated to guaranty the productive efficiency of the pastures.

Key words: Grass, Mulato II, Marandú, decumbens, Piatá, Xaraés, leaf area, sheet, stem, root. 


\section{Introducción}

Del total de tierra de uso agrícola en el Ecuador (11 659087 ha), el $29 \%$ (3 425412 ha) está cubierto con pastos cultivados y el 12 \% (1 $385549 \mathrm{ha}$ ) con pastos naturales. Del total de tierra dedicada a pastos cultivados, el 30 \% (1 038066 ha) está en la Sierra, $45 \%$ en la Costa ( 1533418 ha) y $25 \%$ en el Oriente ( 853928 ha), mientras que del total de pastos naturales, el $75 \%$ se encuentra en la Sierra (1 041461 ha), el $17 \%$ en la Costa (237 246 ha) y el $8 \%$ en el Oriente(106 842 ha) (INEC, 2012). En las zonas tropicales del país, la mayoría de los pastos sembrados son variedades tradicionales como saboya (Panicum maximun) y especies de Brachiaria como B. humidicola, B. brizantha y B. decumbens (CAIZ, 2002).

Para el establecimiento de praderas en el trópico húmedo, se deben escoger especies de pastos de crecimiento rápido, que puedan competir eficientemente con el desarrollo agresivo de las malezas (Lemus, 2008; Faría, 2006), toda vez que las pasturas que se establecen rápidamente pueden soportar mejor las fluctuaciones climáticas a través del año, que controlan el rendimiento y calidad de los forrajes (Romero, 2006). El periodo más critico se presenta en la época seca, donde por falta de agua existe escasez de pasturas para los animales y el material disponible es de baja calidad (Sosa et al., 2005). Por estas razones, se considera que la etapa más delicada en la producción de forrajes es la fase del establecimiento y por esto es importante la selección de la especie adecuada con características que le permitan superar las limitantes ambientales (Faría, 2005).

En la actualidad se han liberado al mercado diferentes genotipos del género Brachiaria como mejores opciones para la producción de forrajes en climas adversos (Faría, 2006). Dentro de estos materiales se destacan Mulato II, Marandú, Piatá y Xaraés reportadas como especies forrajeras de alto valor nutricional, que crecen en diversos tipos de suelo y que son resistentes y/o tolerantes a plagas y enfermedades que afectan la productividad y persistencia de otros pastos (Argel et al., 2007).
Algunas de estas variedades de brachiaria presentan características agronómicas como la rápida emergencia de los tallos y una alta tasa de crecimiento lo que facilita el establecimiento de las pasturas (Olivera et al., 2006; Olivera et al., 2008). De esta manera, el crecimiento inicial constituye una fase crítica en el desarrollo de la planta porque en esta etapa se genera y se expande el sistema radicular que soporta el crecimiento de la parte aérea y ello determina la capacidad de la planta para competir con las malezas y aprovechar adecuadamente los recursos ambientales (Romero et al., 2005). Un análisis de crecimiento relaciona las condiciones morfológicas de la planta a lo largo del ciclo fenológico y se puede utilizar para determinar el efecto del ambiente sobre el crecimiento de la planta, competencia con las malezas y las diferencias genotípicas de capacidad productiva (Magalhães, 1986; Stefanini et al., 2002).

La presente investigación tuvo como objetivo de evaluar el crecimiento de cinco variedades de Brachiaria durante las primeras 12 semanas de crecimiento después de la emergencia de la plantas.

\section{Materiales y métodos}

El proyecto de investigación se condujo en el periodo comprendido entre abril y junio del 2012 en la Granja Experimental El Oasis, perteneciente a la Escuela de Ingeniería Agropecuaria de la Universidad Tecnológica Equinoccial, Extensión Santo Domingo. El sitio experimental se encuentra localizado en el $\mathrm{km} 4$ de la carretera a San Jacinto del Búa a $00^{\circ}$ 13’37" de latitud Sur y 79² 15’04” de longitud Oeste. El clima prevalente en la zona se clasifica como tropical húmedo, con temperatura media anual de $23.5{ }^{\circ} \mathrm{C}$ y precipitación anual de 2,600 a 2,800 mm distribuida en seis meses de invierno y seis de verano (Miranda, 2010).

Para el trabajo experimental se utilizaron 300 fundas negras de polietileno, con una capacidad aproximada de $2 \mathrm{~kg}$, que fueron llenadas con suelo de la granja al cual se añadieron suficientes nutrientes, 
previo análisis del suelo, para asegurar el normal crecimiento de las plantas. Se procedió luego a la siembra por semilla de las diferentes braquiarias en las fundas, con evaluaciones semanales, iniciándose el muestreo a los 7 días posteriores a la emergencia de la plántula. Las fundas con las plantas se distribuyeron por genotipo en el sitio experimental que estuvo ubicado a la intemperie. Se utilizó como testigo la especie Brachiaria decumbens para compararla con tres variedades mejoradas de Brachiaria brizantha (Marandú, Xaraés y Piatá) y con el híbrido Mulato II.

Los muestreos fueron tipo destructivo, separando manualmente hojas (lamina foliar + vaina), tallos y raíz. Para cada una de estas partes de la planta se hicieron estimaciones de la materia verde (MV) y seca (MS) acumuladas en cada período. Las variables a evaluar fueron altura de planta, largo de la raíz y tallo, área foliar, peso fresco y peso seco de la raíz, tallo y hojas, y el área foliar se determinó utilizando la metodología empleada por Rincón et al. (2012)

Para el análisis estadístico se utilizó un modelo de mediciones repetidas en el tiempo, en un diseño completamente al azar con cinco repeticiones:

$$
\mathrm{Y}_{\mathrm{ijk}}=\mu+\tau_{\mathrm{i}}+\delta_{\mathrm{ij}}+\rho_{\mathrm{k}}+(\tau \rho)_{\mathrm{ik}}+\varepsilon_{\mathrm{ijk}}
$$

Donde: $\mathrm{Y}_{\mathrm{ijk}}=$ Variable de respuesta; $\mu=$ Media General; $\tau_{\mathrm{i}}=$ Efecto de la i-ésima variedad; $\delta_{\mathrm{ij}}=$ Error asociado a las unidades experimentales; $\rho_{\mathrm{k}}=$ Efecto del muestreo; $(\tau \rho)_{\mathrm{ik}}=$ Efecto de la Interacción entre las variedades y el muestreo y $\varepsilon_{\mathrm{ijk}}=$ Error Aleatorio. Se realizó una prueba de comparación de medias con Tukey con $\alpha=0.05$.

La eficiencia fisiológica de la planta se determinó por medio del crecimiento en función del tiempo. Para hacer este tipo de análisis se utilizaron dos mediciones básicas, la producción en materia seca de la fracción aérea del material vegetal y el área foliar (Carranza et al., 2009). Los parámetros utilizados para estimar la eficiencia fisiológica de la planta fueron índice de área foliar (IAF), área foliar específica (AFE), tasa de asimilación neta (TAN), tasa de crecimiento del cultivo (TCC) y tasa relativa de crecimiento (TRC). El índice de área foliar (IAF) es un valor adimensional que expresa el área foliar (AF) de la planta en relación al área que ocupa el suelo (Hunt, 1990), y se calcula con la siguiente relación:

$$
\mathrm{IAF}=\frac{\mathrm{AF}_{\text {planta }}}{\mathrm{A}_{\text {suelo }}}
$$

El área foliar específica (AFE) se define como la relación entre el área foliar de la planta y el peso seco de la hoja (Hunt, 1990) y se calcula usando la siguiente fórmula:

$$
\mathrm{AFE}=\frac{\mathrm{AF}_{\text {planta }}}{\mathrm{W}_{\text {Hoja }}}
$$

La tasa de crecimiento del cultivo (TCC), ó tasa absoluta de crecimiento (TAC), es el incremento de la materia seca de la planta o de cada uno de sus órganos por unidad de tiempo (Hunt, 1990) y se calcula de la siguiente forma:

$$
\mathrm{TCC}=\frac{\mathrm{W}_{2}-\mathrm{W}_{1}}{\mathrm{~T}_{2}-\mathrm{T}_{1}}
$$

La tasa de asimilación neta (TAN) expresa la eficiencia fotosintética de la planta y se define como el incremento en el peso de la materia seca por unidad de área foliar por unidad de tiempo (Aguilar-García et al., 2005; Tayeb, 2012) y decrece con la edad de la planta. Se calcula de la siguiente forma:

$$
\mathrm{TAN}=\frac{\mathrm{W}_{2}-\mathrm{W}_{1}}{\mathrm{~T}_{2}-\mathrm{T}_{1}} * \frac{\log A \mathrm{~F}_{2}-\log A \mathrm{~F}_{1}}{A \mathrm{~F}_{2}-A \mathrm{~F}_{1}}
$$

Donde W1 y W2 indican el peso de la materia seca en tiempo, T1 y T2 son unidades de tiempo y AF son las respectivas áreas foliares.

\section{Tsafiquiligrasen}


La tasa relativa de crecimiento (TRC) se define como el incremento en la biomasa por unidad de tiempo en función de la biomasa existente y no es constante (Hunt et al., 2002; Tayeb, 2012). Se calcula de la siguiente forma:

$$
\mathrm{TCR}=\frac{1}{\mathrm{~W}} * \frac{\mathrm{W}_{2}-\mathrm{W}_{1}}{\mathrm{~T}_{2}-\mathrm{T}_{1}}
$$

\section{Resultados y discusión}

\section{Efecto de la edad en los genotipos de braquiarias}

A las 12 semanas se obtuvieron los mayores valores $(P<0.05)$ (Cuadro 1), para altura de planta, longitud de raíz, número de hojas e hijuelos por planta, área foliar, peso del tallo, hoja y raíz; lo cual concuerda con lo observado por Avellaneda et al. (2008), quienes evaluaron tres genotipos de braquiarias, y observaron que los tallos aparecieron a partir de la semana cuatro, y la relación hoja/tallo disminuyó con la edad, lo cual es un comportamiento típico en las braquiarias (Avellaneda et al., 2008; Pérez et al., 2004). Se encontró que en las primeras tres semanas las plantas mostraron un crecimiento acelerado del sistema radicular y fotosintético, esto se da porque planta en los primeros días de vida debe competir por la absorción de agua y nutrientes, además de la captación de la radiación solar (Lucchesi, 1984; Clavero y Urdaneta, 1997).

\begin{tabular}{|c|c|c|c|c|c|c|c|c|c|}
\hline \multirow[b]{2}{*}{ Semanas } & \multicolumn{8}{|c|}{ Variable } & \multirow[b]{2}{*}{$\mathrm{h} / \mathrm{t}^{9}$} \\
\hline & Altura ${ }^{1}$ & Raíz ${ }^{2}$ & Hojas $^{3}$ & Hijuelos $^{4}$ & $\begin{array}{c}\text { Área } \\
\text { Foliar }^{5}\end{array}$ & Tallo $^{6}$ & Hoja ${ }^{7}$ & Raiz $^{8}$ & \\
\hline 1 & $9.20 \mathrm{a}$ & $5.10 \mathrm{a}$ & $1.00 \mathrm{a}$ & * & $5.82 \mathrm{a}$ & * & $0.02 \mathrm{a}$ & $0.01 \mathrm{a}$ & * \\
\hline 2 & $14.30 \mathrm{~b}$ & $14.70 \mathrm{~b}$ & $2.00 \mathrm{ab}$ & $*$ & $18.27 \mathrm{bc}$ & * & $0.07 \mathrm{ab}$ & $0.03 \mathrm{a}$ & * \\
\hline 3 & $18.60 \mathrm{c}$ & $22.74 \mathrm{c}$ & $2.84 \mathrm{~b}$ & $1.40 \mathrm{~b}$ & $36.05 \mathrm{~cd}$ & * & $0.13 \mathrm{ab}$ & $0.06 \mathrm{a}$ & $*$ \\
\hline 4 & $23.98 \mathrm{~d}$ & $36.45 \mathrm{~d}$ & $2.92 \mathrm{~b}$ & $1.55 \mathrm{~b}$ & $57.60 \mathrm{e}$ & $0.01 \mathrm{~B}$ & $0.28 \mathrm{bc}$ & 0.18 at & b $\quad 28.0$ a \\
\hline 5 & $25.98 \mathrm{~d}$ & $41.36 \mathrm{e}$ & $3.32 \mathrm{~b}$ & $2.72 \mathrm{cb}$ & $93.13 \mathrm{f}$ & $0.02 \mathrm{~B}$ & $0.41 \mathrm{c}$ & 0.24 at & $20.5 \mathrm{~b}$ \\
\hline 6 & $34.08 \mathrm{e}$ & $43.40 \mathrm{f}$ & $6.08 \mathrm{c}$ & $3.40 \mathrm{c}$ & $236.49 \mathrm{~g}$ & $0.06 \mathrm{Bc}$ & $0.90 \mathrm{~d}$ & $0.42 \mathrm{c}$ & $15.0 \mathrm{c}$ \\
\hline 7 & $46.36 \mathrm{f}$ & $45.70 \mathrm{~g}$ & $9.04 \mathrm{~d}$ & $3.96 \mathrm{c}$ & $314.90 \mathrm{~h}$ & $0.13 \mathrm{C}$ & $1.68 \mathrm{e}$ & $0.95 \mathrm{~d}$ & $12.9 \mathrm{~d}$ \\
\hline 8 & $52.24 \mathrm{~g}$ & $48.98 \mathrm{~h}$ & $13.24 \mathrm{e}$ & $4.60 \mathrm{c}$ & $468.10 \mathrm{i}$ & $0.29 \mathrm{D}$ & $3.07 \mathrm{f}$ & $2.07 \mathrm{e}$ & $10.6 \mathrm{e}$ \\
\hline 9 & $57.64 \mathrm{~h}$ & $51.32 \mathrm{i}$ & $16.56 \mathrm{f}$ & $5.32 \mathrm{~cd}$ & $660.80 \mathrm{j}$ & $0.46 \mathrm{E}$ & $3.97 \mathrm{~g}$ & $2.54 \mathrm{f}$ & $8.6 \mathrm{f}$ \\
\hline 10 & $69.76 \mathrm{i}$ & $57.50 \mathrm{j}$ & $20.20 \mathrm{~g}$ & $6.28 \mathrm{~d}$ & $921.60 \mathrm{k}$ & $0.75 \mathrm{~F}$ & $5.29 \mathrm{~h}$ & $3.33 \mathrm{~g}$ & $7.1 \mathrm{f}$ \\
\hline 11 & $75.36 \mathrm{j}$ & $60.84 \mathrm{k}$ & $27.60 \mathrm{~h}$ & $8.00 \mathrm{e}$ & 1334.401 & $1.64 \mathrm{G}$ & $8.39 \mathrm{i}$ & $5.35 \mathrm{~h}$ & 5.1 \\
\hline 12 & $81.16 \mathrm{k}$ & 65.121 & $38.24 \mathrm{i}$ & $12.64 \mathrm{f}$ & $1793.80 \mathrm{~m}$ & $2.58 \mathrm{H}$ & $11.15 \mathrm{j}$ & $10.29 \mathrm{i}$ & $4.3 \mathrm{~h}$ \\
\hline P Variedad & $<0.001$ & $\underset{0.001}{<}$ & $<0.001$ & $<0.01$ & $<0.001$ & $<0.001$ & $<0.001$ & $<0.001$ & $<0.001$ \\
\hline
\end{tabular}

Cuadro 1. Efecto de la edad en el crecimiento inicial de las braquiarias

\section{Efecto de los genotipos de Brachiaria en el crecimiento inicial}

Al comparar las cinco brachiarias evaluadas en el estudio, se observó que la variedad Piatá presentó mayor altura de planta ( $\mathrm{P}<0.05)$ que el híbrido Mulato II, desde el inicio del crecimiento. Esto concuerda con lo observado por Argel et al. (2007), quienes mencionan que el hibrido tiene menor altura en comparación a otras variedades de brizantha. Este comportamiento previene el volcamiento o acame en zonas con vientos fuertes.

Al contrario, cuando se evaluó el largo de raíz se observó que Mulato II presentó mayor longitud radicular $(\mathrm{P}<0.05)$ desde el inicio de la evaluación (Figura 1) y que la variedad Xaraés fue la de menor longitud con respecto a las demás variedades $(\mathrm{P}<0.05)$. La capacidad de la plata para desarrollar raíz es una característica que puede ayudar a que la planta se establezca mejor, ya que una raíz larga permite explorar mejor el suelo y esto incrementa la capacidad de la planta para absorber agua y nutrientes (Clavero y Urdaneta, 1997). Esta es una característica deseable en los forrajes para que puedan competir efectivamente con las malezas y aprovechen adecuadamente los recursos ambientales (Faría, 2005). Si bien el híbrido Mulato II presentó una altura menor que las demás variedades, su capacidad de desarrollar raíces de mayor longitud podría incrementar su habilidad para establecimiento.

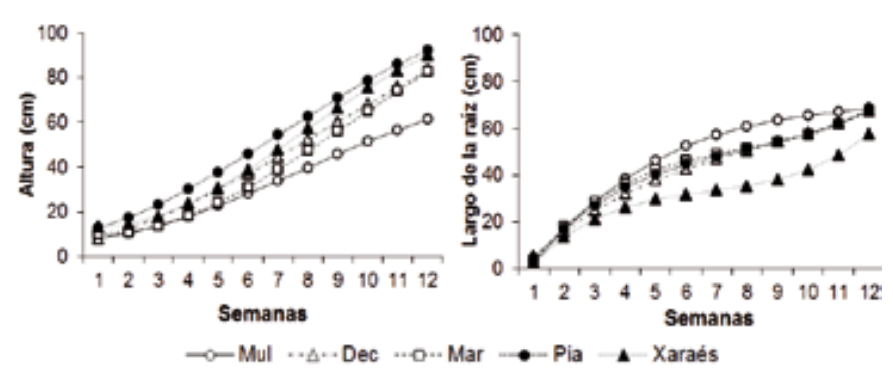

Figura 1. Comportamiento en la altura de la planta y longitud de la raíz durante el crecimiento inicial en cinco variedades del género Brachiaria sembradas en la época de lluvias. 
De los genotipos evaluados, tanto decumbens como Piatá fueron las más precoces ya que presentaron tallos en la cuarta semana, mientras que en Marandú, Xaraés y Mulato II lo hicieron hasta la octava (Figura 2). Cabe señalar también que se observó mayor acumulación de materia seca en tallo $(P<0.05)$ en aquellas que el tallo se desarrolló a partir de la cuarta semana en comparación con las que lo hicieron en la octava. En la semana 12 el peso del tallo $(P<0.05)$ fue de 11.98, 11.68, 12.76, 7.13 y 6.39g para decumbens, Piatá, Marandú, Mulato y Xaraés, respectivamente. Sin embargo, la híbrido Mulato II fue superior a decumbens ( 9.49 vs. $6.90 \mathrm{~g}$ ) en peso seco de raíz $(\mathrm{P}<0.05)$, pero inferior a la Piatá, Marandú y Xaraés (13.36, 10.88 y 10.78 , respectivamente). Esto le daría a Mulato II ventaja en establecimiento en relación a decumbens, ya que esta última presentó una menor masa radicular, pero no con respecto a las demás edades, por tener menor capacidad de exploración del suelo.

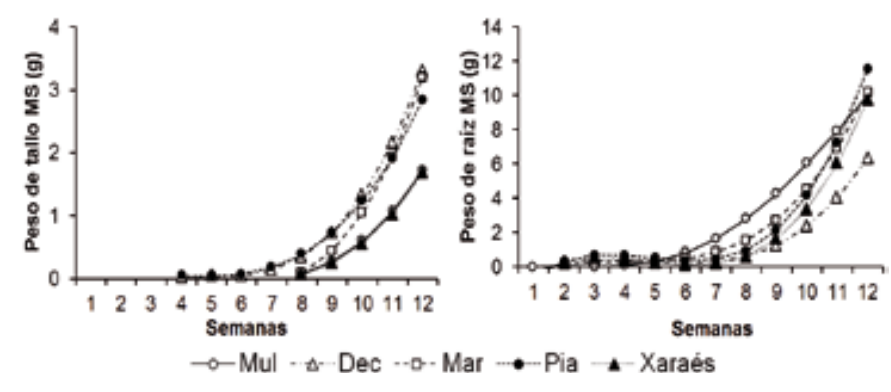

Figura 2. Comportamiento en el peso de tallos y de raíz en las primeras 12 semanas de crecimiento posteriores a la emergencia de la planta.

En cuanto al número de hijuelos por planta, se observó una tendencia semejante a la de longitud de raíz entre los genotipos ( $P>0.05)$ durante las doce semanas en estudio. Sin embargo, Mulato II presentó mayor número de hijuelos que la decumbens $(\mathrm{P}<0.05)$ desde la primer semana de evaluación (Figura 3) hasta la 11, mientras que las variedades Piatá y Xaraés mostraron un comportamiento semejante, con menor número de hijuelos que las demás ( $P>0.05)$. El número de hijuelos es un indicador agronómico muy importante en la producción de forrajes ya que determina la capacidad de macollamiento y cobertura de los pastos (Jimba y Adedeji, 2003). De las evaluaciones hechas se puede establecer que el hibrido Mulato II presentó una mejor capacidad de macollamiento y cobertura a la semana 11, situación que no ocurrió con Xaraés y Piatá. En cuanto al número de hojas, la variedad decumbens presentó el mayor número $(\mathrm{P}<0.05)$ en la semana 12 (Figura 3) en comparación con las otras variedades evaluadas. Es importante enfatizar el hecho de que en la semana uno y dos todas las variedades desarrollaron el mismo número de hojas y solamente a partir de la tercer semana del crecimiento se observó un comportamiento diferente $(\mathrm{P}<0.05)$ en todas las variedades.

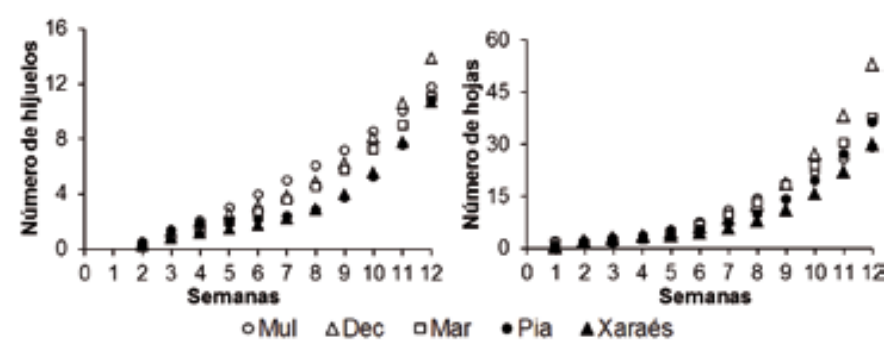

Figura 3. Numero de hijuelos y hojas en las primeras 12 semanas de crecimiento posteriores a la emergencia de la planta.

La variedad Marandú presentó mayor acumulación de peso seco en hoja (vaina + lamina foliar) $(P<0.05)$, mientras que Mulato II, Xaraés y Piatá fueron iguales ( $P>0.05)$, pero diferentes a decumbens $(P<0.05)$ (Figura 4). Cabe señalar que a pesar de que la decumbens presentó un mayor número de hojas, éstas tuvieron un menor peso en la masa foliar ya que son de menor tamaño. Esto puede ser un indicador de la calidad del forraje producido. En la Figura 4 también se puede observar el cambio del área foliar a través del tiempo en los cinco genotipos evaluados, observándose que decumbens presentó la menor área foliar y Marandú, Mulato II y Xaraés obtuvieron los valores de mayor magnitud, lo que les da mayor capacidad para la captación de la luz solar. 


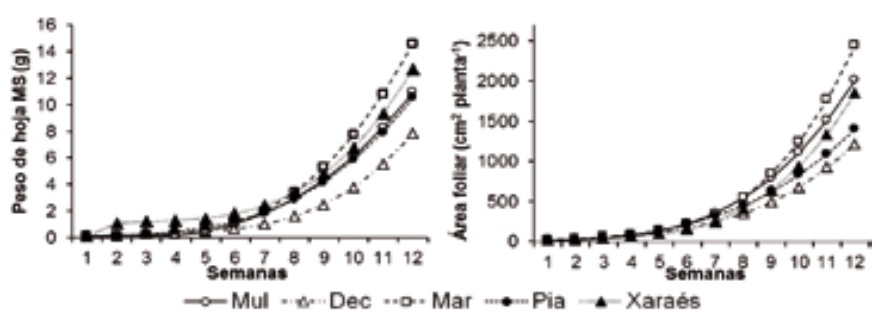

Figura 4. Comportamiento del peso de hojas y área foliar de la plantas en las primeras 12 semanas de crecimiento posteriores a la emergencia.

Al evaluarse la eficiencia fisiológica de la planta de los cinco genotipos, se observó que el índice de área foliar (IAF) se incrementó con el paso de tiempo (Figura 5). En la semana 12 la variedad Marandú presentó el mayor IAF, seguida de Mulato II y Xaraés, mientras que la variedad decumbens presentó el menor valor.

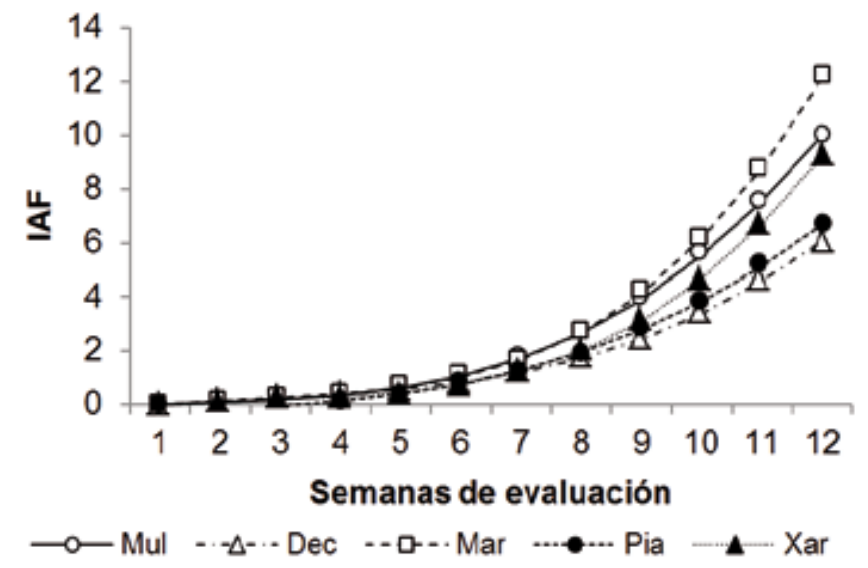

Figura 5. Índice de área foliar en las primeras 12 semanas de crecimiento posteriores a la emergencia.

En términos generales, el área foliar específica (AFE) de los genotipos disminuyó con la edad (Figura 6), debido a que con el paso del tiempo se incrementó el espesor de la hoja (Witkowski y Lamont, 1991). Los resultados mostraron que Mulato II presentó una mayor AFE, lo que indica que sus hojas fueron más delgadas que las variedades de Piatá y Xaraés.

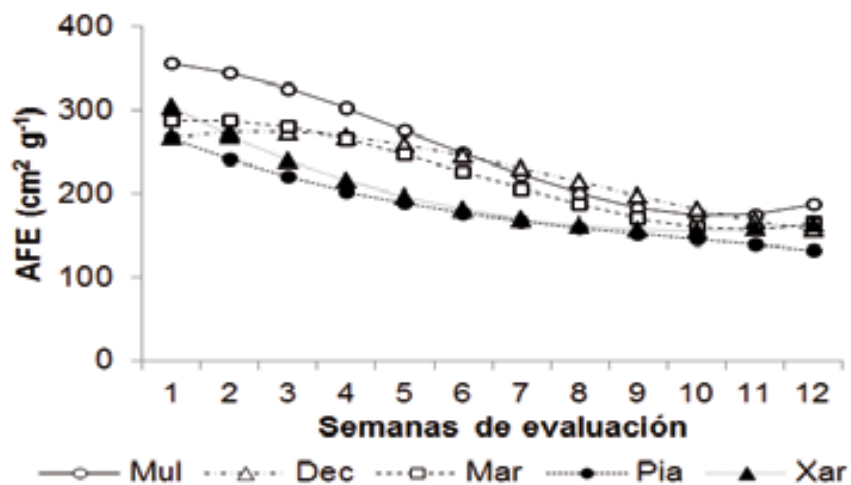

Figura 6. Área foliar especifica (AFE) en las primeras 12 semanas de crecimiento posteriores a la emergencia.

La tasa de crecimiento del cultivo (TCC) de los genotipos evaluados también aumentó con la edad (Figura 7), lo cual es un indicador de que la división celular en los tejidos meristemáticos causaron una intensiva e irreversible expansión de células (Carranza et al., 2009). Se destacó el Marandú por tener la TCC más alta desde el inicio de la evaluación, lo que se traduce en una mayor acumulación de biomasa por unidad de área, mientras que la variedad de Piatá fue la que presentó menor TCC.

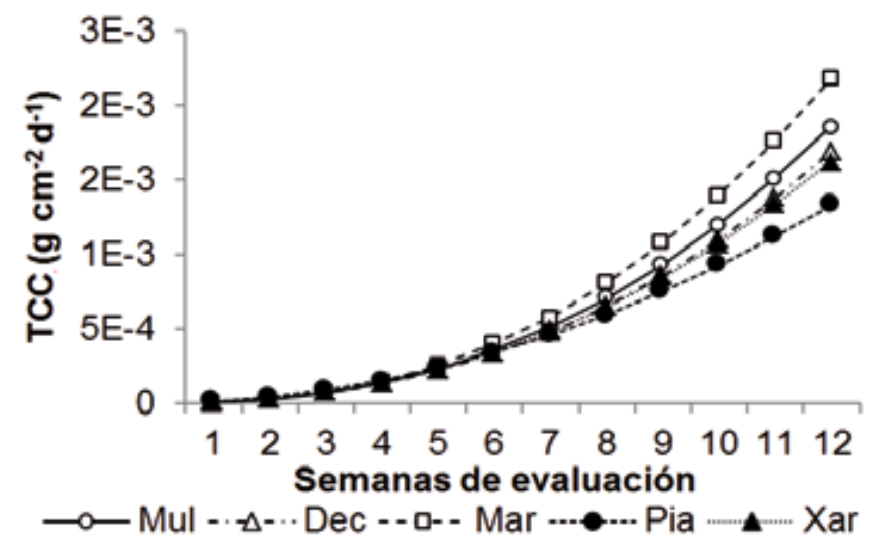

Figura 7. Tasa de crecimiento del cultivo (TCC) en las primeras 12 semanas de crecimiento posteriores a la emergencia.

Para todos genotipos evaluados, la tasa de asimilación neta (TAN) disminuyó con el paso del tiempo (Figura 8), demostrando un 
comportamiento típico en este parámetro en los pastos (Del Pozo et al., 2001; Hoyos et al., 2009). Cabe señalar que el hibrido Mulato II presentó la menor TAN, debido probablemente a la menor altura y alta área foliar. Estás dos condiciones hacen que las hojas se encuentran sobrepuestas lo que reduce la TAN como consecuencia de la menor intercepción de la radiación solar (Carranza et al., 2009). En el caso de Piatá, el comportamiento fue contrario al de las demás variedades y presentó mayor altura y un área foliar baja lo que permitió que las hojas fueran más eficientes en la utilización de la radiación solar por estar menos traslapadas.

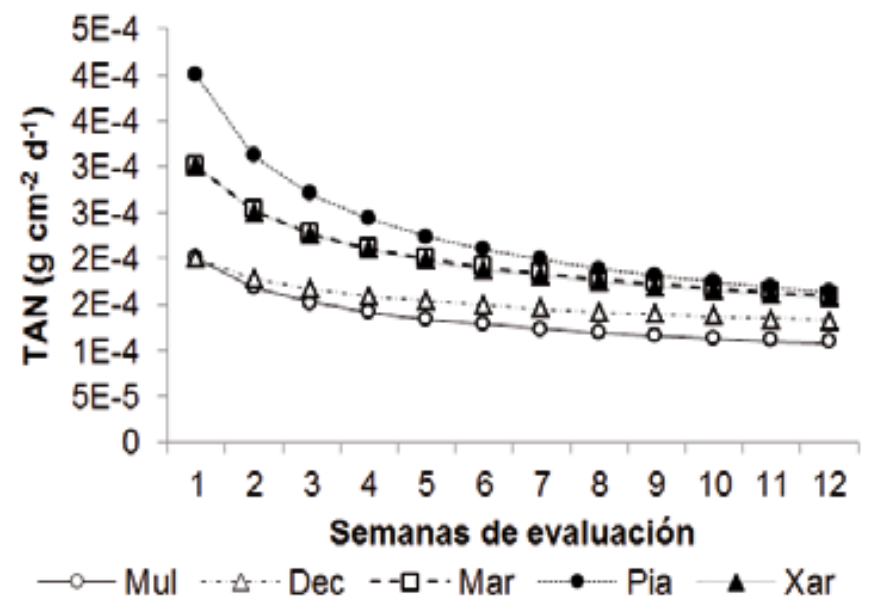

Figura 8. Tasa de asimilación neta (TAN) en las primeras 12 semanas de crecimiento posteriores a la emergencia.

Para todos los genotipos evaluados, la tasa relativa de crecimiento (TRC) se redujo con el paso del tiempo (Figura 9), observándose que a medida que la planta creció, la TRC se redujo debido a la existencia de una menor producción de fotoasimilados en relación a biomasa seca existente (Hoyos et al., 2009).

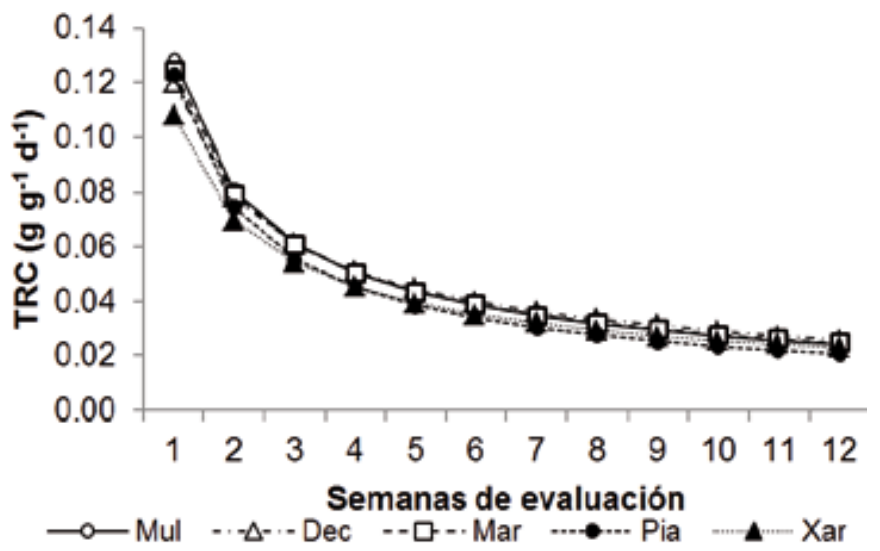

Figura 9. Tasa relativa de crecimiento (TRC) en las primeras 12 semanas de crecimiento posteriores a la emergencia.

\section{Conclusiones}

Los datos acumulados en este estudio demostraron que el comportamiento de los genotipos evaluados fue diferente, existiendo diferencias significativas en la dinámica de crecimiento inicial y la eficiencia fisiológica través del tiempo. Estas diferencias son importantes para la selección de genotipos, ya que de esta elección depende éxito en el establecimiento de las pasturas. En las condiciones en las que se llevó a cabo este experimento, el hibrido Mulato II fue el que presentó los mejores atributos caracterizados por una alta área foliar y un buen crecimiento del sistema radicular en las primeras semanas después de la emergencia. Este genotipo seria una buena opción en la zona para evitar problemas durante el establecimiento. 


\section{Agradecimientos}

Al Consejo Nacional de Ciencia y Tecnología (CONACYT), por la Beca 425135/266100 y la Beca Mixta 290618, que hicieron posible la estancia del primer autor en el Ecuador.

A la Universidad Tecnológica Equinoccial Campus Santo Domingo y a la Facultad de Ingeniería y Ciencias de la Universidad Autónoma de Tamaulipas por las facilidades otorgadas para hacer posible esta investigación.

\section{Bibliografía}

Avellaneda C., J., F. Cabezas G., G. Quintana Z., R. Luna M., O. Montañez V., I. Espinoza G., S. Zambrano M., D. Romero G., J. Vanegas R. y E. Pinargote M. 2008. Comportamiento agronómico y composición química de tres variedades de Brachiaria en diferentes edades de cosecha. Ciencia y Tecnología. 1(2): 87-94.

Aguilar-García, L., J. A. Escalante-Estrada, L. Fucikovsky-Zak, L. Tijerina-

Chávez y E. Mark. 2005. Área foliar, tasa de asimilación neta, rendimiento y densidad de población en girasol. TERRA Latinoamericana. 23(3): 303-310.

Argel, P. J., J. W. Miles, J. D. Guiot, H. Cuadrado y C. E. Lascano. 2007. Cultivar mulato II (Brachiaria híbrido CIAT 36087): Gramínea de alta calidad y producción forrajera, resistente a salivazo y adaptada a suelos tropicales ácidos bien drenados. CIAT. Colombia. $22 \mathrm{p}$.

CAIZ (Cámara de Agricultura de la Primera Zona). 2002. Cámara de Agricultura de la Primera Zona (en línea). Consultado el 21 de junio 2012. Disponible en: http://www.agroecuador.com/ HTML/Censo/censo_4395.htm

Carranza, C., O. Lanchero, D. Miranda y B. Chaves. 2009. Análisis del crecimiento de lechuga (Lactuca sativa L.) Batavia cultivada en un suelo salino de la Sabana de Bogotá. Agronomía Colombiana 27(1): 41-48.

Clavero, T., y R. Urdaneta. 1997. Crecimiento del sistema radical del pasto elefante enano (Pennisetum purpureum cv Mott). Revista de la Facultad de Agronomía-LUZ. 14: 657-663.

Del Pozo, P., R. Herrera, M. García, A. M. Cruz, y A. Romero. 2001. Análisis del crecimiento y desarrollo del pasto estrella con y sin adición de fertilizante nitrogenado. Revista Cubana de Ciencia Agrícola. 35(1): 51-58.

Faría M., J. 2005. Establecimiento de pasturas. En: González, C. y Soto, E. (eds). Manual de ganadería doble propósito. Astro Data S.A. Maracaibo, Venezuela. pp. 156-161.

Faría M., J. 2006. Manejo de pastos y forrajes en la ganadería de doble propósito. En: X Seminario manejo y utilización de pastos y forrajes en sistemas de producción animal. Maracaibo, Venezuela. pp.1-9.

Hoyos, V., M. Rodríguez, J. F. Cárdenas-Hernández y H. E. BalagueraLópez. 2009. Análisis del crecimiento de espinaca (Spinacia oleracea L.) bajo el efecto de diferentes fuentes y dosis de nitrógeno. Revista Colombiana de Ciencias Hortícolas. $3(2): 175-187$.

Hunt, R. 1990. Basic growth analysis: Plant growth analysis for beginners. Unwin Hyman. London, England. $111 \mathrm{p}$.

Hunt, R, D. R. Causton, B. Shipley and A. P. Askew. 2002. A modern tool for classical plant growth analysis. Annals of Botany. 90:485-488.

INEC (Instituto Nacional de Estadísticas y Censos). 2012. Visualizador de estadísticas Agropecuarias del Ecuador ESPAC para el año 2010. Consultado el 20 de Abril del 2012.

http://www.inec.gov.ec/estadisticas/?option=com_content\&view=ar ticle\&id $=103 \&$ Itemid $=75$ 
Jimba, S. C., and A. A. Adedeji. 2003. Effect of plant spacing in the nursery on the production of planting materials for field establishment of vetiver grass. Tropicultura. 21(4): 199-203.

Lemus, R. 2008. Guidelines for Pasture Establishment. Forage News. $4: 1-8$

Lucchesi, A. 1984 .Utilização prática da análise de Crescimento vegetal. Anais da Escola Superior de Agricultura Luiz de Queiroz. 41(1):181:202.

Magalhães, A. 1986. Análise quantitativa de crescimento. In: Fisiologia Vegetal. Ferri, M. EDUSP, Brasil. pp:331-350.

Miranda, K. 2010. Agenda zonal para el buen vivir, propuestas de desarrollo y lineamientos para el ordenamiento territorial. Monsalve Moreno. Ecuador. $95 \mathrm{p}$.

Olivera, Y., R. Machado y P. P del Pozo. 2006. Características botánicas y agronómicas de especies forrajeras importantes del género Brachiaria. Pastos y Forrajes. 29(1):1-13.

Olivera, Yuseika et al. 2008. Persistencia del pastizal en una colección de Brachiaria spp. en un suelo ácido. Pastos y Forrajes. 31 (4):333.

Pérez, J. A., E. García, J. F. Enríquez, A.R. Quero, J. Pérez y A. Hernández. 2004. Análisis de crecimiento, área foliar específica y concentración de nitrógeno en hojas de pasto “mulato” (Brachiaria híbrido, cv.). Técnica Pecuaria en México. 42(3): 447-458.

Romero, O. 2006. Estacionalidad en la producción de forrajes. Tierra Adentro. 71:36-39

Stefanini, M. B., S. D. Rodrigues, e L. C. Ming. 2002. Ação de fitorreguladores no crescimento da erva-cidreira-brasileira. Horticultura Brasileira. 20(1):18-23.

Tayeb, S. 2012. Calculate \& Analyze of Growth in Vicia faba L. Plant. Life Science Journal. 9(1):850-852.

Witkowski, E. T., and B. B. Lamont. 1991. Leaf specific mass confounds leaf density and thickness. Oecologia. 88:486-493. 
